\title{
Metamodels Resulting from Two Different Geometry Morphing Approaches Are Suitable to Direct the Modification of Structure-Born Noise Transfer in the Digital Design Phase
}

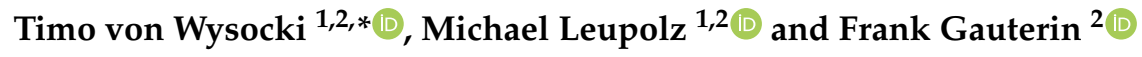 \\ 1 Mercedes-Benz AG, 70372 Stuttgart, Germany; michael.leupolz@yahoo.de \\ 2 Karlsruhe Institute of Technology, Institute of Vehicle System Technology, 76131 Karlsruhe, Germany; \\ frank.gauterin@kit.edu \\ * Correspondence: timo.von_wysocki@daimler.com
}

Received: 13 October 2020; Accepted: 31 October 2020; Published: 6 November 2020

\begin{abstract}
Noise vibration and harshness (NVH) development often takes place in the later development phases. Shifting the optimization to the early digital development phase enables more parameters to participate in the optimization and leads to a more holistic development process. Digital NVH development often modifies system and component frequency response functions (FRFs) using finite element (FE) simulation. Currently, the often manual process of creating new FE models for modified designs makes a systematic evaluation of many designs difficult and time-consuming. In this paper, we take on these difficulties and use both a Direct Morphing approach and a Box Morphing approach to automatically adopt the first existing FE models to modified designs. We use the generated simulation results to fit metamodels describing the correlation between geometrical parameters and characteristic FRF values. These metamodels provide an easy and fast to use tool for designers to consider NVH demands. In a simulation example, we demonstrate the capabilities by modifying the kinematic hard points of a vehicle suspension and using them to modify the noise transfer sensitivity. We show that the metamodels can lead the digital design process to intuitively and specifically reduce characteristic component FRF values by changing the location of the component hard points.
\end{abstract}

Keywords: morphing; optimization; metamodel; NVH; FEM; interpolation; transfer function; optislang; ANSA; python

\section{Introduction}

In the vehicle development process, noise, vibration and harshness (NVH) are becoming more important [1,2]. Often, NVH improvements take place late in the development process [2], when the product design is mostly fixed [3] (p. 4). This limits the possibilities to improve NVH performance. A more holistic approach considers NVH design in the earlier, digital development phases [4].

In concept phases, when hardware is not available yet, NVH design relies on finite element (FE) simulation. The used FE models originate from meshing CAD geometry with often manual interference. Thus, each design iteration can necessitate time-consuming remeshing and rebuilding the FE model, which slows down concept evaluation [3]. One approach to resolve these issues is the usage of morphing technologies, where the FE model of a predecessor or similar component is adapted to the new shape by morphing the FE geometry [3].

Amplitude reduction of Frequency Response Functions (FRFs) is an established method to improve $\mathrm{NVH}$ performance as they determine the transfer of structure-born noise through the 
component. The component FRFs are part of the system transfer behavior from vibration source to receiver.

In this paper, we modify FRFs of a vehicle suspension component by changing its geometry in the early, digital design phase. We use a Direct Morphing-inspired and a Box Morphing approach to automatically generate changed geometries originating from an initial FE geometry. We compare both approaches regarding effort, time and results. Both approaches generate a large data base of simulation results which is used to create metamodels to describe geometry influences on FRF characteristics. These metamodels offer a simple tool in the design phase to determine directions of design changes. Finally, we use an automated procedure to adjust and optimize the component's FRFs, eigenfrequencies or stiffness by changing the component's geometry. This approach enables a first $\mathrm{NVH}$ optimal design in the early, digital design phase and preserves later hardware testing to fine-tune NVH performance.

The structure of this paper is presented in Figure 1. In Sectionsec:sota, we present the state of the art concerning $\mathrm{NVH}$ development in early design phases and morphing approaches. In Section 3 , we introduce our approach. First, we present both morphing approaches; second, the usage of metamodels. In Section 4, we perform a study on a component of a vehicle suspension in which we reduce the transferred force at a specific frequency, using our approach. In this section, we compare both morphing approaches and their application.

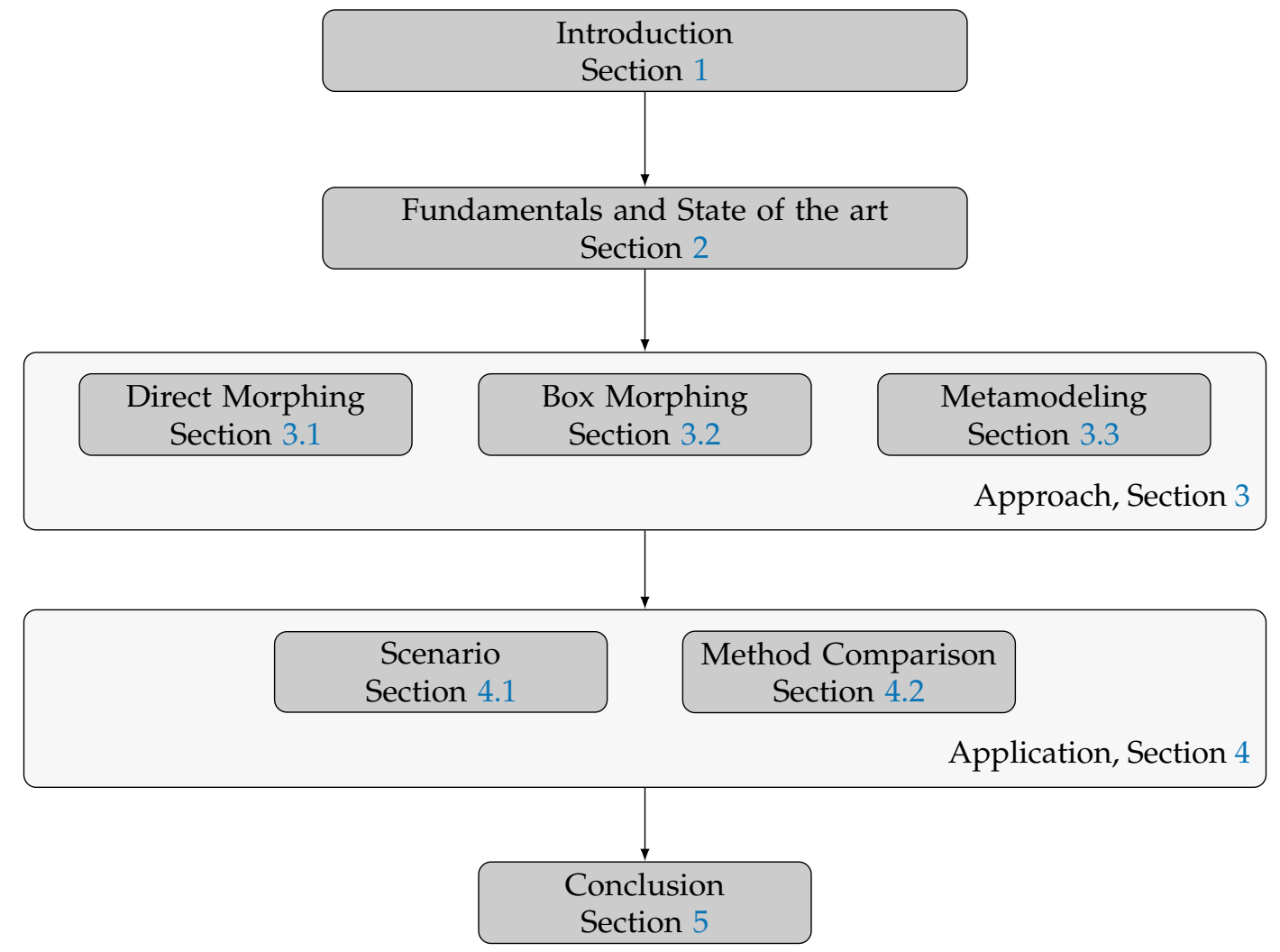

Figure 1. Structure of this paper.

\section{Fundamentals and State of the Art}

In this section, we briefly present the state of the art concerning NVH optimization in the digital design phase and the fundamentals of different morphing approaches.

\subsection{NVH Optimization in the Digital Design Phase}

In the early design phase, $\mathrm{NVH}$ optimization relies on simulation, as no hardware is available yet [5]. Nowadays, evaluations are based on three different simulation model origins: CAD models of recent generations, predecessor FE models or new simulation models from scratch [3] (p. 2), [6] 
(p. 1653), [7] (p. 4180). The first method requires a parametric CAD model to allow fast modifications [4] (p. 27). However, each design change still has to be meshed in order to receive a new FE model [8] (p. 170). This slows down the development process [3]. The second method, using old FE models, requires a method to alter the existing FE model to resemble the new concept under consideration. One of these methods is morphing, where an algorithm distorts the existing FE mesh to approximate the desired design. As this process is done in the digital design phase, the main target of these generated simulation models is to roughly approach $\mathrm{NVH}$ demands in the design process, not to predict the behavior as accurately as possible [4] (p. 30), [9] (p. 28). In this paper, we use this approach to receive modified FE models. The third method, creating new simulation models from scratch, is time-consuming and requires manual activity for each design variation [3].

\subsection{Morphing}

As this paper focuses on morphing FE models in the digital design phase, we give a brief overview over morphing techniques with special application on FE meshes. Morphing is also called mesh warping or mesh moving in the literature [10] and originates from animations or special effects, where meshes change from a source to a target shape [3]. In this paper, we do not go into mathematical detail, as there are many specific publications on this topic, for example [11].

Volumetric FE meshes consist of nodes that define volumetric elements like e.g., tetrahedrons or hexahedrons [11]. We call this set of elements, forming the component, FE mesh. Besides volumetric elements, there are also 1D and 2D FE elements like beams or shells. We do not mention them separately here, but all used methods in this paper apply to them, too.

Morphing FE meshes usually only moves its nodes in space [3] (p. 4), without changing the element connectivity [6] (p. 1653). As long as the mesh quality stays inside defined boundaries, no remeshing, mesh relaxation or smoothing is necessary [3,12]. This is especially problematic, when morphing distances become large or areas with different morphing directions collide. Due to that, a constant monitoring of mesh quality criteria is required [3]. Those include for example distortion on element level or smoothness and cross sections on component level [3] (p. 21), [8] (p. 307), [13] (p. 22).

Moreover, [11] uses morphing to find the transition process from an initial to a target 3D model. For our application, only the resulting 3D model is relevant, not the transition. Nevertheless, the feature preservation described by [11] (p. 180) is necessary in our approach, too. His example of the feature preservation directly transfers to technical features like screw holes or bushings.

In order to control the morphing process, there are three types of nodes: control nodes, free nodes and fixed nodes [3] (p. 6). The second type receives different names in literature, for example deformable nodes. As we deform the mesh but not the node itself, we prefer the term free.

In literature, there are mainly two different approaches for morphing FE meshes with varying naming. For example, [3] distinguish between Direct Morphing and a control-based approach. In this paper, we use both methods and explain our implementations in Sections 3.1 and 3.2 respectively. We compare their application and results in Sections 3 and 4 respectively. The Direct Morphing approach is slightly altered here. We label our two approaches Direct Morphing and Box Morphing.

\section{Application of Morphing}

Morphing techniques serve many different applications and purposes. The following examples are relevant for our work and thus presented here.

In automotive applications, [3] investigate the influences of the roof curvature, the windshield angle, the wheel base and the subframe shape on eigenfrequencies, airborne and structure-born noise. These are large-scale investigations on a full vehicle level, contrary to the approach presented in this paper, where we focus on component level. They use their control block morphing model as a direct input into the optimization chain, which differs from the approach presented in this paper using metamodels. 
Similarly, [14] perform an investigation on a body in white. They use and compare morphing approaches to modify connection elements between 1D FE elements. They use NX NASTRAN's SOL200 [15] to optimize geometry parameters [14].

Furthermore, [12] use a morphing approach to implement geometric imperfections onto an FE mesh. This enables them to perform a robustness evaluation of the geometrical tolerances on brake squeal and vibration transfer through the knuckle [12,16]. In another publication, [13] use a statistical approach to approximate random brake pad designs in order to investigate robustness [13]. They change FE nodes according to identified distribution parameters [16].

In addition, [17] use the morphing of single nodes to generate beads on larger metal sheets modeled with the use of FE shell elements. This is used to optimize the interior noise in a vehicle passenger cabin [17].

Moreover, [18] use Direct Morphing to optimize the design of a crankshaft to fit the applied forces best and reduce stress inside the component.

\subsection{Morphing Alternatives}

Morphing is not the only approach to investigate concept performance in the digital phase. Topology optimization generates new geometries on a mesh level depending on predefined load cases. Morphing techniques however, change an existing design into a new shape to investigate the influence on specific criteria.

FE models can consist of beam-elements. Especially for thin beam-like structures, this is an easy way of modeling geometries parametrically. Furthermore, [4] use beam-modeling to perform cross-section optimization [4]. Moreover, [14,19] use beams to investigate body modifications. In addition, [9] use beam modeling for twist-beam axles, as these concepts cannot be calculated without FE calculation [9]. As many components do not consist of thin beam-like structures, these approaches are not applicable onto complex shapes in general.

\section{Method}

As illustrated in Figure 2, our approach builds on a given component FE model. Whether this is the model of a predecessor or a completely new FE model is insignificant. For our use case, we are especially interested in the influences of moving the component's kinematic hard points at the connection points to neighboring components. Here, we use both a Direct Morphing-inspired (Section 3.1) and a Box Morphing (Section 3.2) approach to investigate the design changes systematically. In the practical application, one of the methods can be selected. The received simulation data are used to create metamodels that evaluate kinematic hard point location design choices and lead to fast decisions. The hard point locations can thus serve as optimization parameters for the component's FRFs. By splitting simulation and optimization, it is possible to quickly change optimization targets without recalculating the FE simulation [20]. Additionally, the used metamodels can direct design choices without numerical optimization.

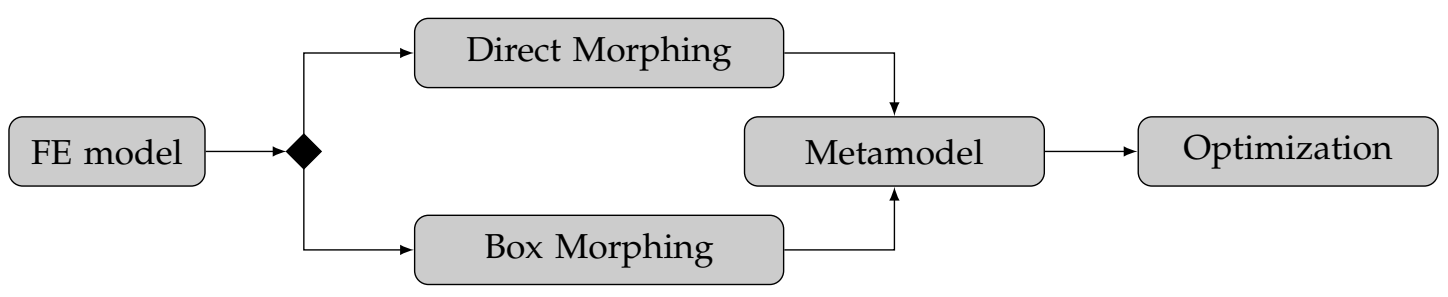

Figure 2. Workflow from initial finite element (FE) model to optimization.

\subsection{Direct Morphing Approach}

Each Direct Morphing process consists of three subprocesses, shown in Figure 3. First, we define the nodes for the three different node types, mentioned in Section 2.2. Control nodes are points in 
space, that impose a given displacement, free nodes are nodes of the FE mesh that are free to move and fixed nodes are points in space that stay at their locations during the morphing process. Next, we apply the actual morphing algorithm onto the set of free nodes and receive their new coordinates. This is done separately for the three directions $x_{1}, x_{2}$ and $x_{3}$. This results in the preliminary morphed mesh. Mesh quality criteria indicate where node relaxation or remeshing is necessary in a last step, resulting in the final morphed mesh.

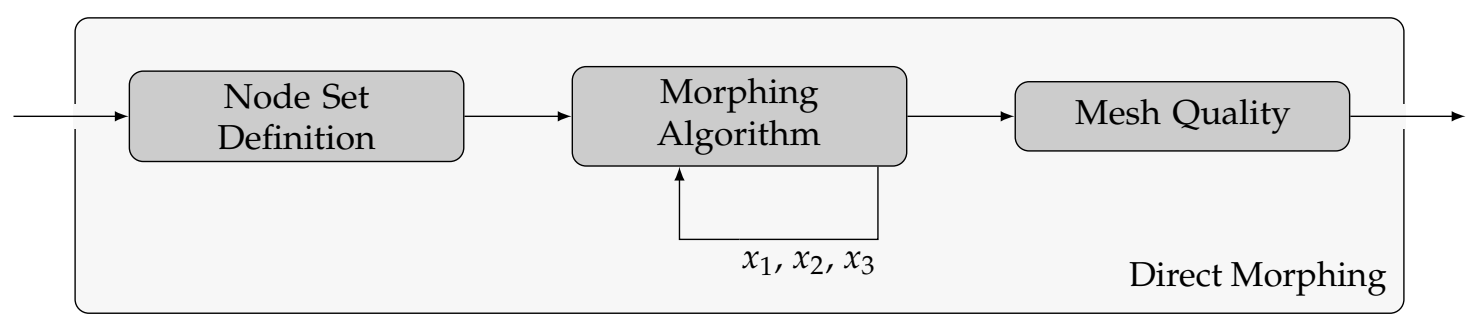

Figure 3. Detailed Direct Morphing process from Figure 2.

For the morphing step, we use the tool chain we proved working in an earlier publication [21] in a slightly modified version. Moreover, [10] suggest using linear morphing and hypothesize natural neighbor as a method, possibly resulting in better element quality by using control nodes in the natural neighborhood [10] (p. 297). In our application, we are confronted with control nodes close to each other, so we needed a method that interpolates independently from the distance to control nodes. We decided to use a combined approach, as [22] suggests as an extension for his approach [22]. Our morphing approach thus consists of two separate interpolation algorithms. First, we generate a three dimensional regular grid of nodes that encases the complete component. We perform a natural neighbor interpolation from the control and fixed nodes onto this grid. Second, we interpolate from this grid onto the actual free FE nodes.

In the following, we describe the morphing algorithm. We use bold symbols to label vectors and underlined symbols to label matrices. Our examples are situated in a global Cartesian coordinate system with the directions $x_{1}, x_{2}$ and $x_{3}$. The last index in vector components specifies the direction.

For the first process step, we define three matrices for sets of control nodes, free nodes and fixed nodes. As our application demands the investigation of design coordinate changes, we want to define displacements at a non-uniform set of points in space, not only on nodes of the FE mesh. For example, the location coordinate of a bushing lies in its center. As the bushing is pressed into a hole of the component, there is no FE node present at this location.

The $k$ control nodes

$$
\underline{x}_{c}=\left[\boldsymbol{x}_{c, 1}, \boldsymbol{x}_{c, 2} \ldots, \boldsymbol{x}_{c, k}\right]=\left[\left(\begin{array}{l}
x_{c, 1,1} \\
x_{c, 1,2} \\
x_{c, 1,3}
\end{array}\right),\left(\begin{array}{l}
x_{c, 2,1} \\
x_{c, 2,2} \\
x_{c, 2,3}
\end{array}\right), \ldots,\left(\begin{array}{l}
x_{c, k, 1} \\
x_{c, k, 2} \\
x_{c, k, 3}
\end{array}\right)\right]
$$

are points in space, defined by their coordinates, independent from the FE mesh. Each control node receives an imposed displacement in the form of a displacement vector, originating from the corresponding control node. This yields a second matrix for the control nodes

$$
\underline{v}_{c}=\left[\boldsymbol{v}_{\mathcal{c}, 1}, \boldsymbol{v}_{c, 2}, \ldots, \boldsymbol{v}_{c, k}\right]
$$

containing all the imposed displacements.

The $m$ fixed nodes

$$
\underline{x}_{f}=\left[x_{f, 1}, x_{f, 2}, \ldots, x_{f, m}\right]
$$


are points in space, defined by their coordinates, independent from the FE mesh, too. As these fixed nodes can be seen as control nodes with the displacement vector $v=0=(0,0,0)^{T}$, we combine the control nodes and fixed nodes into one matrix of imposed displacement nodes

$$
\underline{x}_{d}=\left[x_{c, 1}, x_{c, 2}, \ldots, x_{c, k}, x_{f, 1}, x_{f, 2}, \ldots, x_{f, m}\right]
$$

with their displacements

$$
\underline{v}_{d}=\left[\boldsymbol{v}_{c, 1}, \boldsymbol{v}_{c, 2}, \ldots, \boldsymbol{v}_{c, k}, \mathbf{0}, \mathbf{0}, \ldots, \mathbf{0}\right]
$$

All $n$ FE nodes are collected in one matrix and provide the free nodes

$$
\underline{x}_{F E}=\left[\boldsymbol{x}_{F E, 1}, \boldsymbol{x}_{F E, 2}, \ldots, \boldsymbol{x}_{F E, n}\right] .
$$

The morphing algorithm calculates their displacement vectors

$$
\underline{v}_{F E}=\left[\boldsymbol{v}_{F E, 1}, v_{F E, 2}, \ldots, v_{F E, n}\right]
$$

depending on their locations $\underline{x}_{F E}$ and the imposed displacements $\underline{v}_{d}$ at the points $\underline{x}_{d}$.

We use the python [24] package discrete Sibson (natural neighbor) interpolation by [23] to interpolate the imposed displacements $\underline{v}_{d}$ at the nodes $\underline{x}_{d}$ onto the nodes of a regular grid. This grid consists of nodes $\underline{x}_{g}$ with the same equidistant spacing $\lambda$ in all three directions $x_{1}, x_{2}$ and $x_{3}$. Each direction of the vector field represents a separate scalar interpolation, following [22].

In the next step each FE node $\underline{x}_{F E}$ receives its displacement by linear interpolation from the regular grid nodes $\underline{x}_{g}$. We used SciPy's regularGrid to perform this interpolation [25]. The grid distancing $\lambda$ was set to $3 \mathrm{~mm}$ and influences morphing accuracy and calculation time in opposite directions. The accuracy increases linearly with the grid density, whereas the calculation time increases cubical. The used component consists of 170,000 FE nodes. The component has an extent of approximately $300 \times 300 \times 300 \mathrm{~mm}$, which leads to $1,000,000$ grid nodes $\underline{x}_{g}$.

The morphing is only exact if the node of the regular grid coincides with the control node. Lower values of $\lambda$ reduce the maximum possible distance between grid node and control node. If the application demands to morph larger areas like bushings, we define multiple control nodes in the area with the same imposed displacement, so the morphing error in the middle becomes zero. If we need to achieve a displacement by using only one control node, the morphing process can be performed iteratively until the morphing error undercuts a defined limit. This is done for the validation in this paper.

Altogether, we performed 3 natural neighbor interpolations for the 3 directions and use them to perform $3 n$ regular grid interpolations. All mentioned steps, necessary to perform the morphing, are fully scripted in Python, making the morphing process completely automated. The only necessary input are the original FE model that represents the matrix $\underline{x}_{F E}$ and a text-file that specifies the two matrices $\underline{x}_{d}$ and $\underline{v}_{d}$. This enables us to batch morph the designs necessary to create a profound metamodel. The creation of one design takes approximately five minutes on an Intel Core i7 quad-core processor of the 6 th generation.

The mesh quality checks are applied directly before the FE calculation. In our application for morphing distances of up to $10 \mathrm{~mm}$, the criteria hinted no necessary mesh refinements.

\subsection{Box Morphing Approach}

The Box Morphing approach is also referred to as free-form-deformation (FFD) and was first introduced by [26]. Other literature uses the term control block morphing [3]; in this paper, we name the method Box Morphing. Other than the Direct Morphing approach, Box Morphing does not act directly on the FE nodes [3]. 
To deform the original geometry, first a box structure must be created. In case the structure only consists out of parallelepipeds, the used approach is the FFD [26]. In the case that more complex volumes, such as e.g., prismatic ones, are used, the approach is called extended-free-form-deformation (EFFD) [27]. Since the nodes defining these volumes are the ones driving the deformation, we refer to them as control nodes.

For parallelepipedic volumes, the control points are the nodes of the box. For more complex structures, additional control points might be added on each edge to change their curvature. The free and fixed nodes can generally be all FE nodes. Fixed nodes are always those outside the deformed morphing box, unless a box is displaced as whole. Then, the included nodes are free nodes. Additionally to that, relevant FE nodes might be defined as fixed nodes so their location stays unchanged. If none of those are defined, the free nodes are all FE nodes included in the deformed morphing box.

The FE nodes of the part are shifted through connecting the movement of the control nodes to the FE nodes. In the Box Morphing approach, a lattice of morphing boxes is constructed. Then, the FE nodes are linked to the surrounding box by calculating their local coordinates $s, t$ and $u$. By moving the control nodes, the FE nodes also move. [26]

The local coordinates of an FE node $x$ can be calculated through

$$
\boldsymbol{x}=\sum_{s=0}^{v} \sum_{t=0}^{w} \sum_{u=0}^{r} c_{s t u} B_{s} B_{t} B_{u}
$$

Hereby, $B_{s}, B_{t}$ and $B_{u}$ are arbitrary basis functions that only depend on the local coordinate shown by their index. The control nodes $\mathbf{c}_{s t u}$ are also numbered consecutively in each local coordinate so that the whole equation describes a linear combination of the control nodes and basis functions [28].

As a basis function, [26] originally used a Bernstein polynomial but referred to other functions as a B-spline [29] being suitable. The whole process is considered a mapping from $\mathbb{R}^{3}$ to $\mathbb{R}^{3}$ [26]. By not only considering the location of the control points before morphing, but also after morphing $\mathbf{c}_{i j k}^{\prime}$ the displacement of each FE node

$$
\Delta x=\sum_{s=0}^{v} \sum_{t=0}^{w} \sum_{u=0}^{r}\left(\boldsymbol{c}_{s t u}^{\prime}-\boldsymbol{c}_{s t u}\right) B_{s} B_{t} B_{u}
$$

is calculated [28].

Since the Box Morphing approach is considered Indirect Morphing, the used control points are not situated on the FE mesh itself, but are defined in the box structure [3].

In the morphing structure created for this paper, all planes of morphing boxes that are relevant for the deformation of the component are oriented parallel to the planes of the global coordinate system. Due to that, shifting one plane in the direction of its normal vector only results in a displacement of the geometry in the same direction, which makes it easy to track the movement.

Since this paper evaluates metamodels achieved through different morphing methods, the morphing process needs to be automated. This is achieved by a tool chain combining the morphing environment in the FE preprocessor ANSA by BETA CAE [30] and Python scripts.

The morphing itself is also automated in a Python script that only needs the displacement at each kinematic hard point of the component as input values. The morphing process is fragmented into the four steps, presented in Figure 4. 


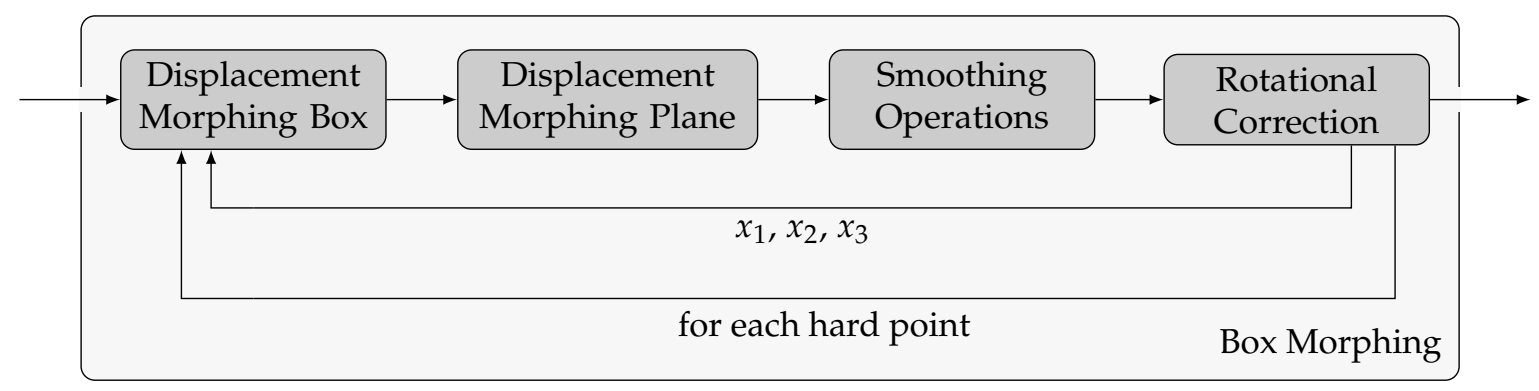

Figure 4. Detailed Box Morphing process from Figure 2.

The first two steps represent the translation of the tracked hard point. The process is partitioned for two reasons. First, the planes being moved are the ones representing the outer layer of the geometry. Using those to clench and stretch the referring area leads to smooth geometries since the deformation in the used tool is linear over the morphing box. To make sure that this does not lead to the transformation of round bearing seats into ovals, the nodes on the bearing seat are defined in a manner that they move in reference to their center point. Second, moving the whole morphing box in addition to one plane leads to a deformation in the surrounding morphing box. That is important to enable larger displacements of the referring point, since the overall deformation is split between multiple boxes through the division in box and plane displacement. Due to that, more FE elements are deformed in the whole process, leading to a smaller deformation of each element itself. For each hard point, an ideal combination between plane and Box Morphing was evaluated empirically.

The third step is necessary to achieve continuous, also described as smooth designs, illustrated in Figure 5.

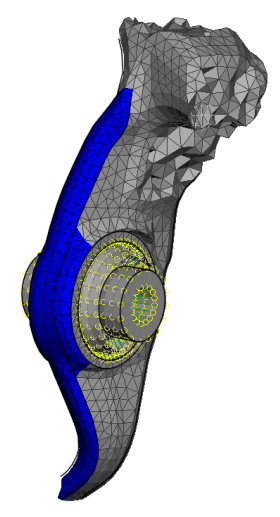

(a)

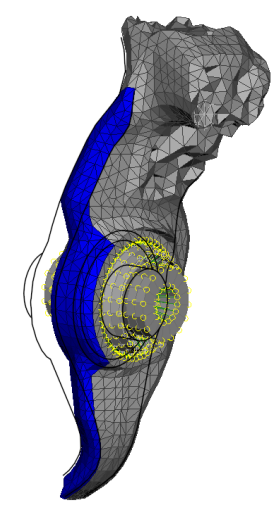

(b)

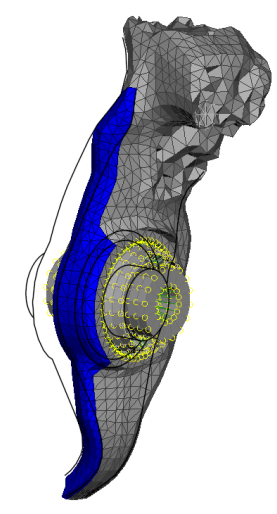

(c)

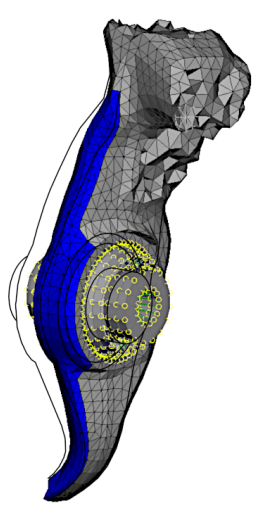

(d)

Figure 5. Visualization of smoothing operation after performing the displacement of one kinematic hard point through Box Morphing. The hard point under investigation is located in the center of the bushing, identified by yellow circles highlighting its nodes. The black outline represents the undeformed geometry in (a). (a) Undeformed. (b) Morphed. (c) 1st smoothing. (d) 2nd smoothing.

The displacement of the hard point is fully realized by moving the morphing box that includes the specific point. This leads to a rather edgy geometry surface which is smoothed by moving two additional sets of control points. Those sets were evaluated empirically and showed satisfying results so that no third smoothing operation is needed. Figure 6 shows all control points being moved to achieve a smooth structure. Figure 6a highlights the control points necessary for the actual displacement. Figure $6 \mathrm{~b}, \mathrm{c}$ highlight the sets of control points moved to achieve a smooth surface.

Sets of control points necessary to smooth the surface of the morphed geometry are defined for each hard point and each dimension. The morphing itself is carried out for each hard point and dimension separately. This is possible since no control point of the constructed box structure affects multiple hard points. Moreover, since all moved planes have normal vectors parallel to one of the 
global axes, they only affect the geometry in that specific direction. In terms of computational effort, this process takes more time than moving all planes in one step. However, since the corresponding plane changes for each direction and hard point, it is necessary to morph them individually. Furthermore, the whole morphing process of one design takes around six minutes if all hard points are moved. We use a virtual machine, running on Intel Xeon 6154.

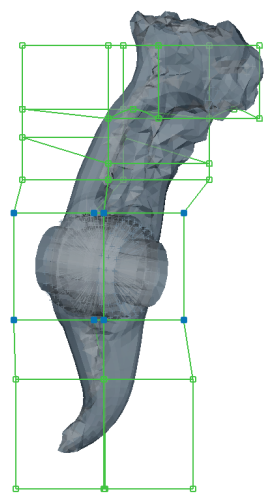

(a)

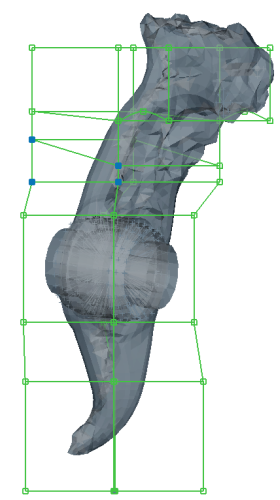

(b)

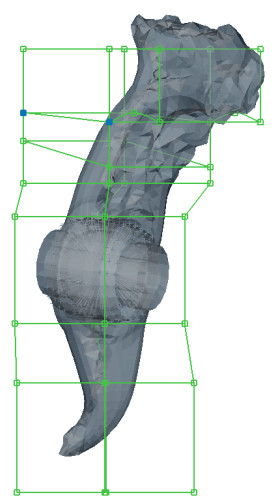

(c)

Figure 6. Showcase of morphing structure necessary for displacement and smoothing operations. (a) Control points for general displacement. (b) Control points for 1st smoothing. (c) Control points for 2nd smoothing.

The last step in Figure 4, the rotational correction, is necessary, as the moving of morphing planes rotates bearing axes or screw holes, if they are not oriented parallel to the moved plane. The described steps fully automate the morphing process.

\subsection{Metamodeling}

Both morphing approaches are completely scripted so the morphing process is automated with an original FE model and text based displacement inputs for the kinematic hard points. This allows us to automatically create a large data base with displacements as input parameters and-in our application-characteristic values of the calculated FRFs as output parameters. This enables us to create an analytical approximation, called metamodel, of the coherence between displacements of hard points and characteristic FRF values.

We create the data base using a space filling latin hypercube approach using Optislang by Dynardo [31]. Depending on the number of geometric input coordinates, we create $N$ designs. The selected morphing approach automatically creates $N$ morphed FE models that are used as input for FE simulation. The morphing error of the Direct and Box Morphing approach is eliminated by using the effective hard point displacements instead of the given ones as input data for the metamodel. From the FRF results we extract characteristic values; for example, the amplitudes $A$ at specific frequencies for specific FRFs of the component:

$$
\underline{A}=\left[A_{1}, A_{2}, \ldots, A_{N}\right]
$$

These values and the input parameters are the basis for a multivariate polynomial regression that predicts the amplitude $\hat{A}$. We use cubic polynomials with coupled terms of the form

$$
\begin{aligned}
\hat{A}(p, q)=\alpha_{0}+\alpha_{1} p+\alpha_{2} p^{2} & +\alpha_{3} p^{3}+\alpha_{4} q+\alpha_{5} p q+\alpha_{6} p^{2} q+\alpha_{7} p^{3} q+\alpha_{8} q^{2} \\
& +\alpha_{9} p q^{2}+\alpha_{10} p^{2} q^{2}+\alpha_{11} p^{3} q^{2}+\alpha_{12} q^{3}+\alpha_{13} p q^{3}+\alpha_{14} p^{2} q^{3}+\alpha_{15} p^{3} q^{3}
\end{aligned}
$$

with two variables $p$ and $q$ and the parameters $\alpha_{0}$ to $\alpha_{15}$. The two dimensional approach enables simple plots that can lead the design process. We use a least squares approach to calculate the parameters $\alpha$ for all combinations of two input parameters and the simulated result value. For example, 
the polynomial $\hat{A}\left(v_{c, 3,1}, v_{c, 3,3}\right)$ describes the regression between the characteristic value $A$ and the imposed displacements $v_{c, 3,1}$ at position 3 in direction 1 and $v_{c, 3,3}$ at position 3 in direction 3.

The coefficient of determination

$$
R^{2}=1-\frac{\sum_{i=1}^{N}\left(A_{i}-\hat{A}_{i}\right)^{2}}{\sum_{i=1}^{N}\left(A_{i}-\bar{A}\right)^{2}}=1-\frac{\sum_{i=1}^{N}\left(A_{i}-\hat{A}\left(p_{i}, q_{i}\right)\right)^{2}}{\sum_{i=1}^{N}\left(A_{i}-\bar{A}\right)^{2}}
$$

with the mean value $\bar{A}$ of the result values $A_{i}$ for the design $i$ of $N$, is an indicator for the quality of the polynomial fit [32] (p. 398). An $R^{2}$ near one hints a good fit.

A combination of well fitted polynomials can then perform as an input for an optimization of $A$. The found optimum is then validated by a separate geometry morph. This procedure fastens optimization as the optimization is performed on an analytical metamodel [20] (p. 231). Additionally, the characteristic parameter $A$ can vary without re-simulating a single design, as $A$ is extracted from the stored results. A changed optimization target and its resulting design can thus be investigated in a matter of minutes.

Additionally to the two dimensional approach, we fit higher dimensional polynomials for numerical optimizations. Again, the optimization parameters can change after performing the FE simulation.

\section{Application}

In this section, we want to use the presented approaches in a practical application and investigate the possibilities and limitations of both morphing approaches and the potential to optimize component FRFs in the digital design phase. We use both morphing approaches to generate data of a component FRF. Using this data, we use simple metamodels to manually affect the component's design and perform a numerical optimization to compare the manual changes to.

\subsection{Scenario}

The application for our approach arises the vehicle chassis development in the digital design phase. We use the first conceptual FE model of a knuckle. This component connects the wheel and the brakes via the suspension links to the car body or the subframe. All structure-born noise from the road and the brake passes the knuckle. We modify the transfer path between wheel hub and suspension links by changing the component's design in order to modify the chassis noise transfer. An illustration of the knuckle is presented in Figure 7.

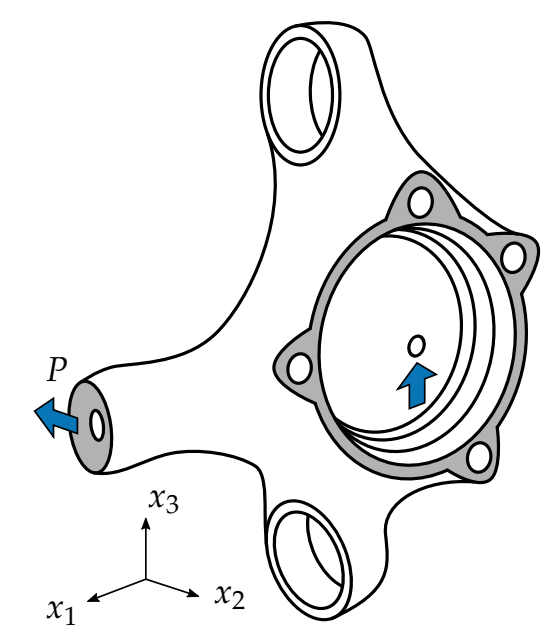

Figure 7. Illustration of the used knuckle. The kinematic hard point $P$ for the connection to the track rod is located in the center of the gray area on the left side. The large gray area on the right represents the connection to the wheel hub. 
In the scenario for this paper, we fix the knuckle at the wheel hub via stiff springs against fixed ground. The stiffness and frequency range is selected in a way; it creates rigid body modes of the knuckle on the springs and elastic modes of the component itself. This represents a simplified test case as it could occur in a full axle. We calculate the FRFs between all the suspension link hard points and the wheel hub force. By changing the location of one of the kinematic hard points to the suspension links, we investigate the influences on the FRFs.

We investigate a displacement of $\pm 10 \mathrm{~mm}$ of the hard point $P$ between knuckle and track rod in three directions. We create 100 designs using space filling latin hypercube by varying the $x_{1}, x_{2}$ and $x_{3}$ coordinates. We call the original coordinate $x_{0}$ and the received design coordinate $x_{D o E}$. We restrict the displacement in the cubical design space to a sphere with $10 \mathrm{~mm}$ radius $r$ and the center point $P$ with the coordinate $x_{0}$. Designs with the Euclidean distance

$$
d=\left|x_{D o E}-x_{0}\right|>r
$$

violate the sphere. These points are shifted towards $P$ to the sphere's surface in order to condense the information on the border. This method follows one of the three suggestions by [20] (p. 221). We calculate the new coordinate on the surface

$$
x_{\text {border }}=x_{0}+\frac{r}{d}\left(x_{D o E}-x_{0}\right) \quad \text { for } \quad d>r .
$$

For the study in this paper, we investigate the FRF between the hard point $P$ for the track rod connection in the direction along the track rod $\left(x_{2}\right)$, marked by the blue arrow on the left in Figure 7 and wheel hub in vertical direction $\left(x_{3}\right)$, marked by the blue arrow on the right. This FRF represents the transfer path of vertical excitation from the road to vibrations induced into the track rod. The FRF is given in Figure 8.

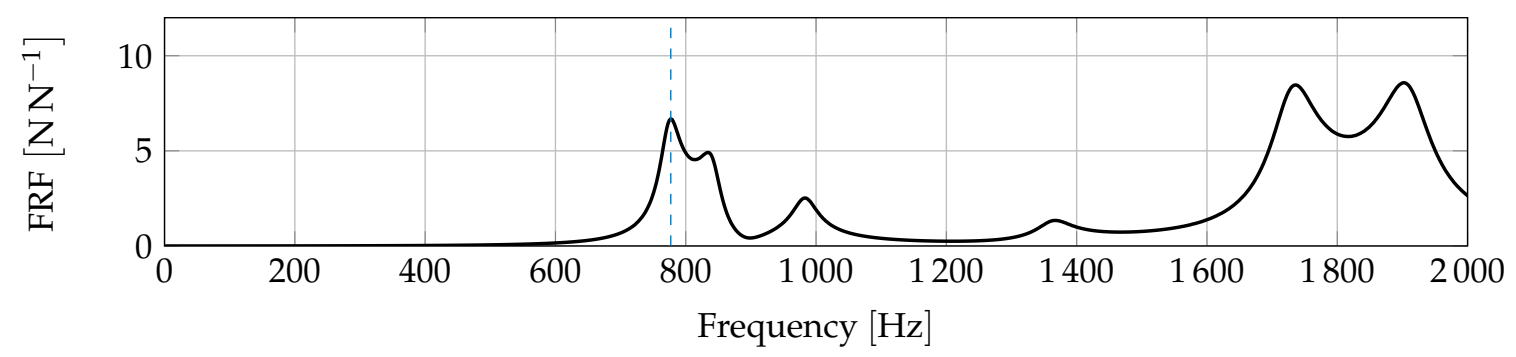

Figure 8. FRF between hard point track rod in $x_{2}$-direction and wheel hub in $x_{3}$-direction. $777 \mathrm{~Hz}$ in blue.

We chose the peak at $777 \mathrm{~Hz}$ with an amplitude of $6.68 \mathrm{~N} \mathrm{~N}^{-1}$ for our investigation. We can reduce the amplitude by two means. We can either reduce the peak's amplitude or change the peak's frequency. The latter is equivalent to changing an eigenmode's frequency $[4,17]$.

In a first step, we compare the FRF range of both morphing approaches for the 100 designs that we generated. In Figure 9 we show the FRF introduced in Figure 8 with an additional Skyline overlay. The gray Skyline indicates the lowest and highest amplitude for all 100 designs. 


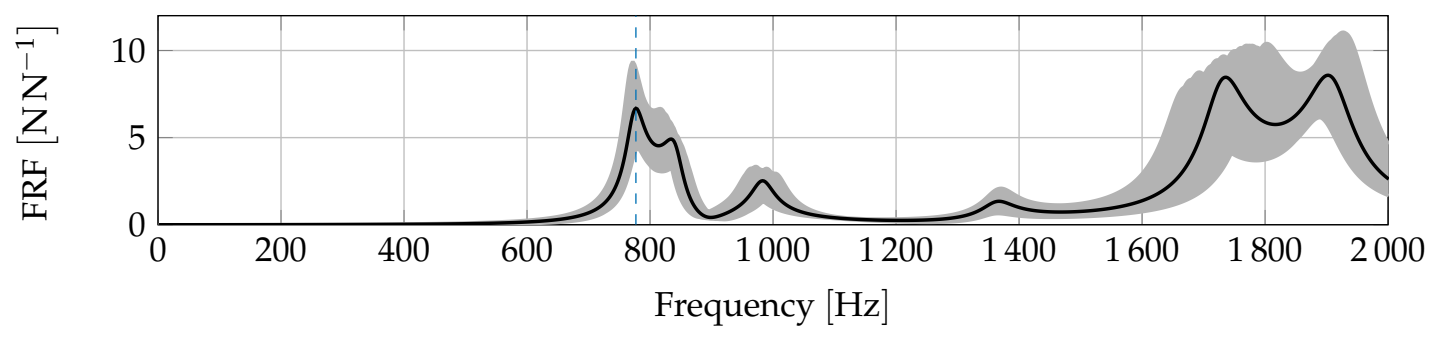

(a)

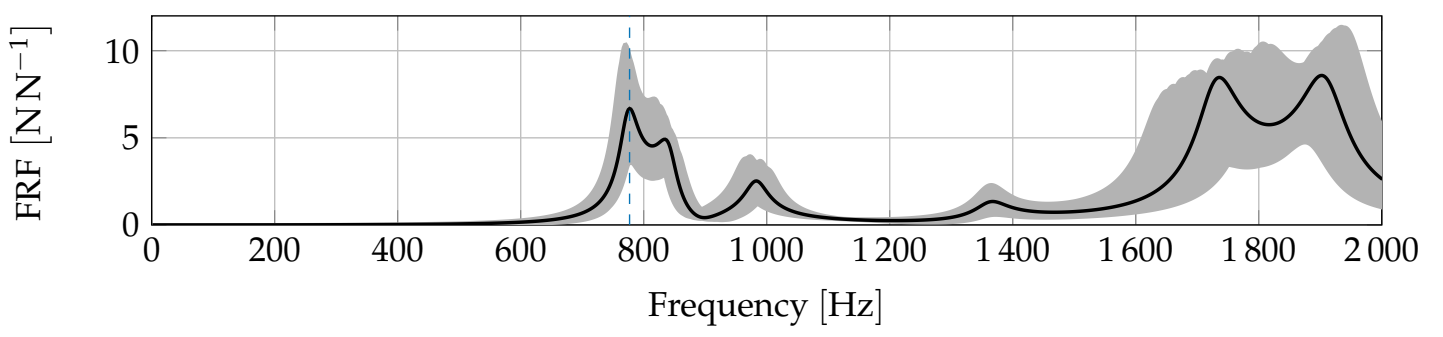

(b)

Figure 9. FRF between hard point track rod in $x_{2}$-direction and wheel hub in $x_{3}$-direction and Skyline of 100 designs. (a) Results using Direct Morphing. (b) Results using Box Morphing.

Moving the hard point by an absolute value of up to $10 \mathrm{~mm}$ around the original attachment point clearly offers a wide variety of changing the FRF, especially in the higher frequency range in which the elastic modes dominate. Both morphing approaches result in similar amplitude bands. Nevertheless, especially at $777 \mathrm{~Hz}$, the maximum and minimum amplitudes of the Skyline vary by $8.5 \%$ and $17.6 \%$ respectively, with the smaller possible changes resulting from Direct Morphing. We discuss the reasons for that after discussing the optimization results. We further investigate whether both data sets result in similar metamodels that describe the underlying phenomena.

Using these 100 designs, we want to receive analytical hints for the NVH optimization in the design process. An easy to use metamodel that takes linked influences into account are polynomials of third order with two variables, introduced in Equation (11). In Figure 10, the two metamodels for Direct and Box Morphing respectively show $R^{2}$ values greater than 0.9 . The original design is located in the center and marked by a black circle.

The amplitude at $777 \mathrm{~Hz}$ clearly depends on all three variables. In Figure $10 \mathrm{~b}, \mathrm{~d}$, we can recognize a direct dependency on the $x_{3}$ coordinate. With varying the $x_{1}$ coordinate, the amplitude hardly changes compared to varying the $x_{3}$-coordinate. The combination of both metamodels indicates an optimum location for $P$ at a small $x_{1}$-value, great $x_{2}$-value and great $x_{3}$-value. The $x_{1}$ - and $x_{2}$ coordinates seem to have smaller influence than the $x_{3}$ coordinate and $x_{1}$ even could stay near $0 \mathrm{~mm}$, depending on the other coordinates. These simple metamodels allow a fast decision for the direction of change, without numerical optimization. We validate them in the next section. 


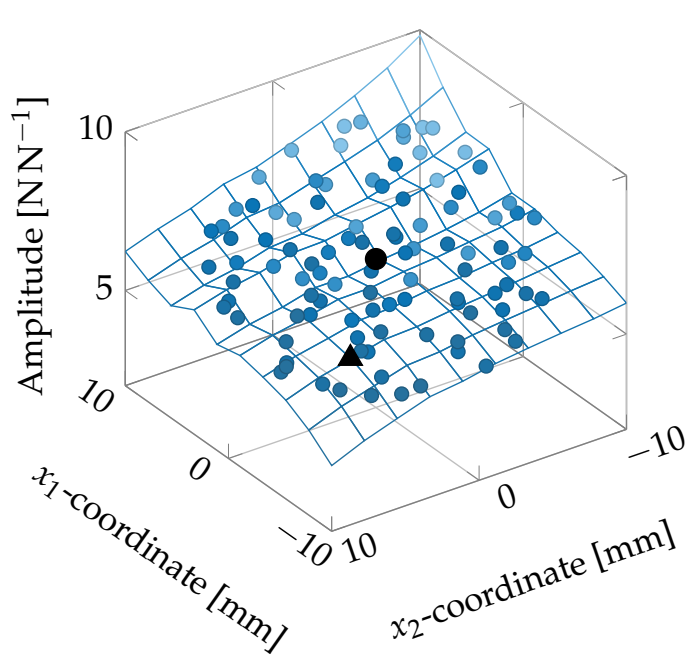

(a)

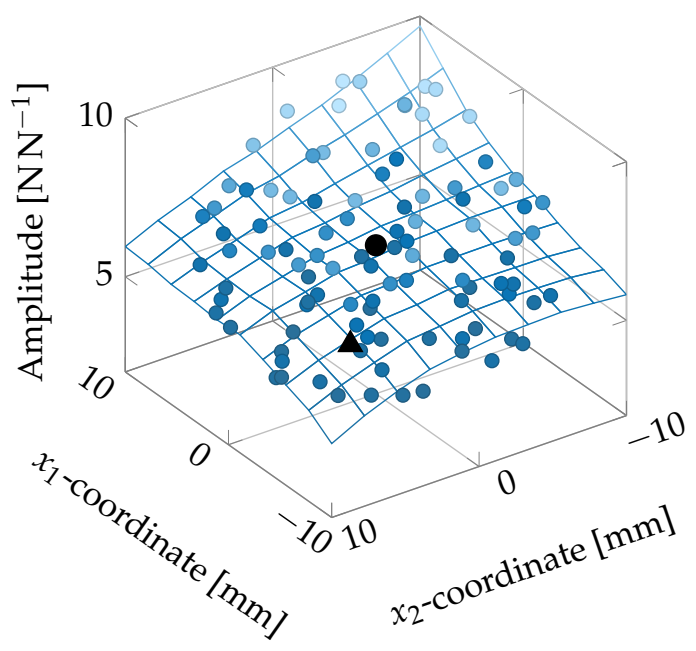

(c)

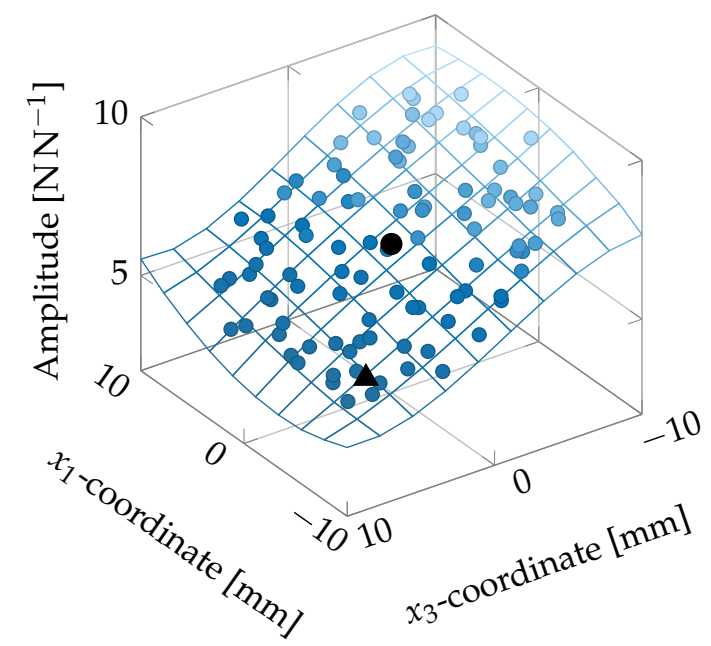

(b)

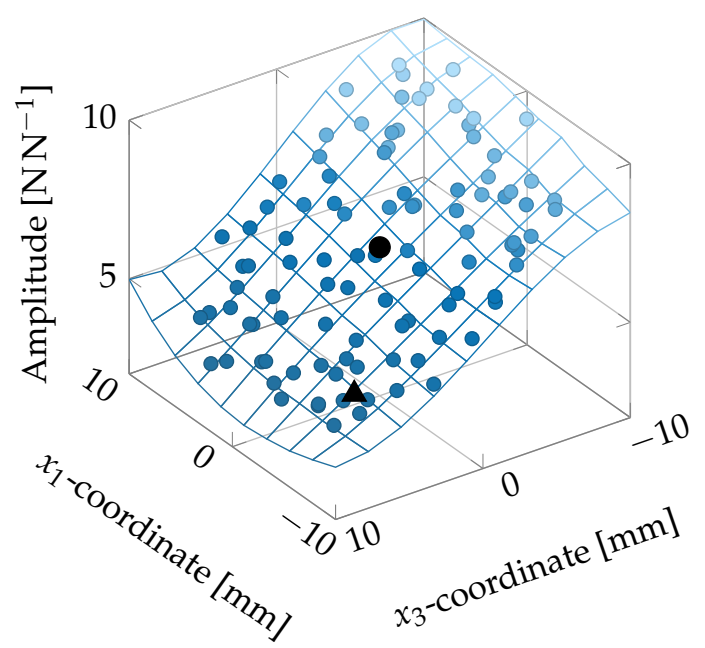

(d)

Figure 10. Metamodels for the influence of the $x_{1}{ }^{-}, x_{2}$ - and $x_{3}$-coordinates derived from 100 design variants by using Direct and Box Morphing. Original design at $(0 \mathrm{~mm}, 0 \mathrm{~mm}, 0 \mathrm{~mm})^{T}$ and an amplitude of $6.68 \mathrm{~N} \mathrm{~N}^{-1}$, highlighted by a black dot. Manually optimized design at $(-5.77 \mathrm{~mm}, 5.77 \mathrm{~mm}, 5.77 \mathrm{~mm})^{T}$, highlighted by a black triangle. (a) Direct Morphing, metamodel for $x_{1}$ - and $x_{2}$-coordinates with $R^{2}=0.94$, manually chosen optimum at $5.8 \mathrm{~N} \mathrm{~N}^{-1}$. (b) Direct Morphing, metamodel for $x_{1}$ - and $x_{3}$-coordinates with $R^{2}=0.90$, manually chosen optimum at $4.7 \mathrm{NN}^{-1}$. (c) Box Morphing, metamodel for $x_{1}$ - and $x_{2}$-coordinates with $R^{2}=0.95$, manually chosen optimum at $5.8 \mathrm{~N} \mathrm{~N}^{-1}$. (d) Box Morphing, metamodel for $x_{1}$ - and $x_{3}$-coordinates with $R^{2}=0.94$, manually chosen optimum at $4.3 \mathrm{NN}^{-1}$.

Polynomials considering all three variables are less vivid, but can lead to better results when using optimization tools. We calculate second order polynomials with three variables and cross terms for both morphing approaches using Optislang. We use a combination of an evolutionary algorithm and a downhill simplex method to find optimum designs. The results using the different models are discussed in the next section. 


\subsection{Method Comparison}

In this section, we compare both morphing approaches and their results for the optimization at $777 \mathrm{~Hz}$. First, we perform a design optimization using the metamodels in Figure 10. As described earlier, we use small $x_{1}$-values, great $x_{2}$-values and great $x_{3}$-values. We use

$$
x_{D o E}=(-10 \mathrm{~mm}, 10 \mathrm{~mm}, 10 \mathrm{~mm})^{T}
$$

and transform it onto the design space surface using Equation (14) and obtain

$$
x_{\text {border }}=(-5.77 \mathrm{~mm}, 5.77 \mathrm{~mm}, 5.77 \mathrm{~mm})^{T}
$$

as the first optimized design. A black triangle represents the design in Figure 10. We create this design using both morphing approaches. The metamodels in Figure 10 hint a resulting amplitude of approximately $5.15 \mathrm{NN}^{-1}$, when averaging the 4 metamodels at the chosen point. Thus the peak is reduced by $23 \%$. The results for the manual and numerical optimization are listed in Table 1 . Both designs resulting from numerical optimization reside on the design space border.

Table 1. Designs, optimized by different metamodels.

\begin{tabular}{ccccc}
\hline Method & $x_{1}$ & $x_{2}$ & $x_{3}$ & Predicted Amplitude \\
\hline Manually & $-5.77 \mathrm{~mm}$ & $5.77 \mathrm{~mm}$ & $5.77 \mathrm{~mm}$ & $5.15 \mathrm{~N} \mathrm{~N}^{-1}$ \\
Optimization Direct & $-4.42 \mathrm{~mm}$ & $3.36 \mathrm{~mm}$ & $8.32 \mathrm{~mm}$ & $4.04 \mathrm{~N} \mathrm{~N}^{-1}$ \\
Optimization Box & $-0.30 \mathrm{~mm}$ & $4.83 \mathrm{~mm}$ & $8.75 \mathrm{~mm}$ & $3.31 \mathrm{~N} \mathrm{~N}^{-1}$ \\
\hline
\end{tabular}

We create all three designs in Table 1 using both morphing approaches and compare the amplitude results and the prediction qualities. The resulting FRFs are shown in Figure 11. The FRF of the original design is marked gray, and the simulation results using Direct and Box Morphing are marked black and blue respectively. The predicted value at $777 \mathrm{~Hz}$ is the amplitude value of the corresponding metamodel.

In Figure 11a, the manually predicted amplitude value from Figure 10 is too high and both morphing approaches hint a stronger amplitude improvement than the two metamodels predict. In Figure 11b,c, the corresponding metamodel matches the simulation result. In each case, the Direct Morphing approach overestimates the amplitude. This is due to the morphing boundary condition for both methods. The Box Morphing approach enforces stronger boundary conditions like bushing axes or machined surfaces to follow the imposed displacements. In contrast, the Direct Morphing approach only enforces single nodes to fit the imposed displacement. Larger imposed areas could be implemented in the Direct Morphing approach, too. Since the method is designed to be fast and easy to use, we decided to impose the displacement at the kinematic hard points only. Figure 11 clearly shows that both morphing approaches detect the same influences, so both methods are suitable to lead design choices. Both the Direct Morphing and the Box Morphing approach proved suitable for the prediction of FRF characteristics. The two-dimensional metamodels provide an easy and fast to use tool to direct design changes using visual hints for improvement. A component designer is able to approximate design changes without numerical calculation or optimization, while still being able to perform an optimization when necessary. The workflow to achieve the predictions and the accuracy differs though. 


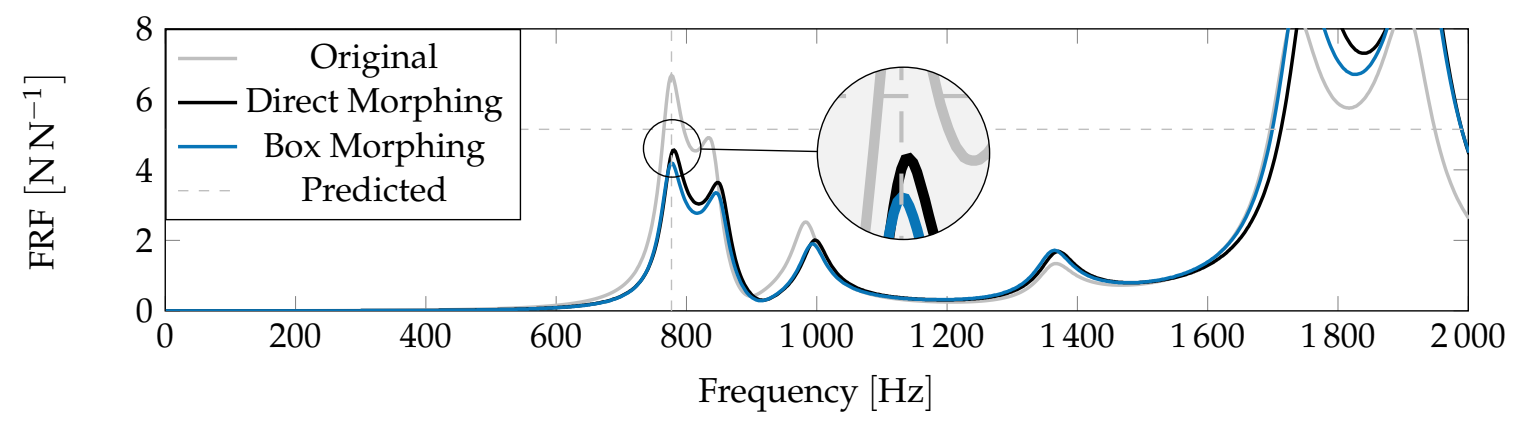

(a)

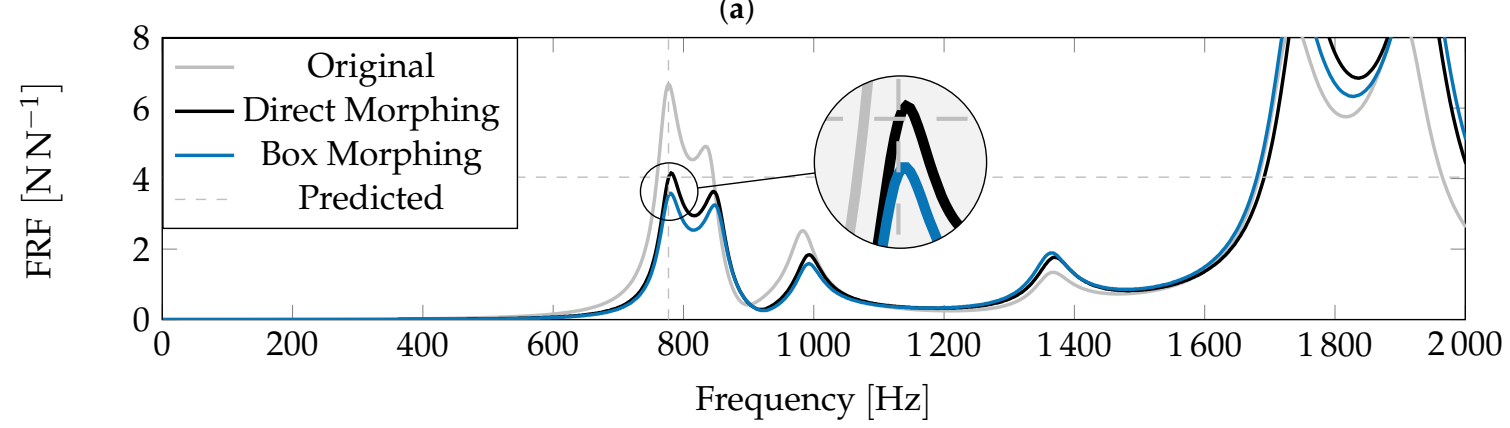

(b)

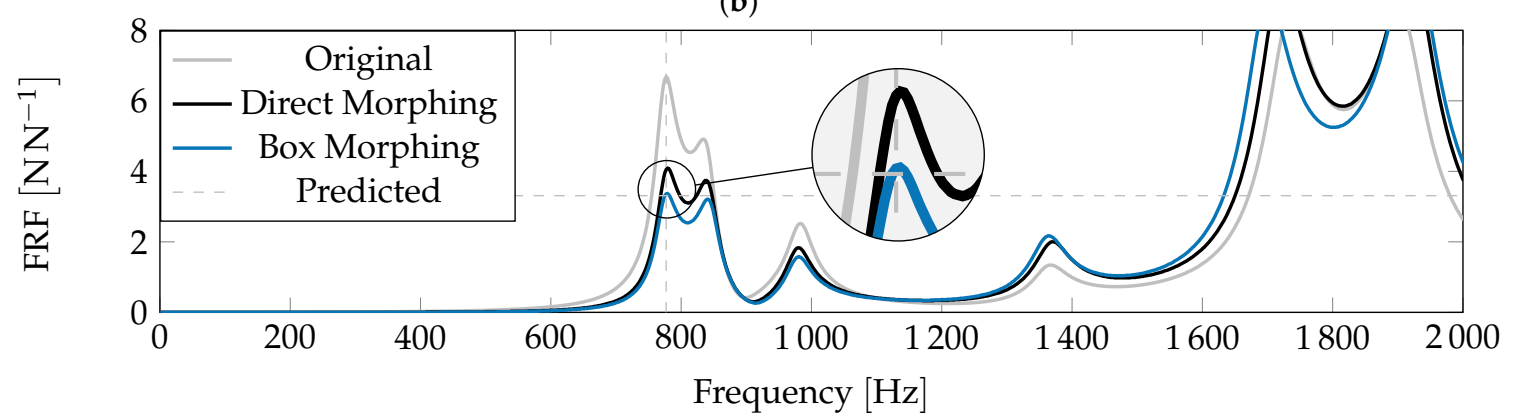

(c)

Figure 11. Three different optimization methods and simulation results obtained by different morphing approaches. (a) Manual Optimization via separate metamodels in Figure 10. (b) Optimization via Optislang metamodel resulting from Direct Morphing data. (c) Optimization via Optislang metamodel resulting from Box Morphing data.

Reusing the tools and workflows created for this paper, predictions for another component are possible. The Direct Morphing approach only needs to receive the FE model of the new component as matrix $\underline{x}_{F E}$ and the new displacement matrices $\underline{x}_{d}$ and $\underline{v}_{d}$. The generation of these matrices takes approximately one working hour and the tool is ready to morph and create metamodels. The Box Morphing approach requires much more manual work. Like [3] observed, the definition of the control boxes is quite time-consuming [3]. Especially for such a complex component as the used knuckle, creating the Box Morphing structure took an estimated time of $100 \mathrm{~h}$. Furthermore, the whole implementation of the code including the identification of splitting factors of plane and Box Morphing as well as smoothing operations took another 100 working hours. That must almost be completely repeated if a different part should be morphed, since the morphing scripts refer to specific control points with values that were evaluated specifically for the used knuckle. Nevertheless, schemes like the rotational corrections might be applied easily on different parts, making at least parts of the morphing script reusable.

The extra effort needed for the generation of the Box Morphing structure comes with greater possibilities to control the morphing behavior. The preservation of axes or surface shapes is easily 
possible in contrast to the Direct Morphing approach. Recent research identifies the Box Morphing approach as less suitable when it comes to how precisely the wanted geometry is achieved than morphing methods that act directly on the FE nodes [28]. Since this paper evaluates the influence of the location of specific hard points on the FRF, only the displacements of these points are relevant. The exact shape of the geometry is less important and unknown, at least in the early design phase. This prevents us from applying an exact shape on the nodes in the Direct Morphing approach.

In our scenario, the averaged deviation for the morphing accuracy of the Box Morphing is $0.02 \%$ after the first morphing iteration. The Direct Morphing approach converges towards the target coordinate, but due to the regular grid it will never reach the coordinate exactly. As the interpolation inside the regular grid is linear though, it is possible to alter the target coordinate so that the morphed shape matches the desired one. With two iterations, we reach an accuracy of $0.2 \%$.

The mesh quality is automatically checked before each FE calculation and does not violate any quality criteria, used for regular FE investigations, neither for the Direct Morphing approach, nor the Box Morphing approach. The limitation to small geometry changes prevents different control nodes from morphing into each other, which would result in erroneous meshes. Visually, the morphed geometries are smooth with preserved features, meaning that we consider the received designs close to designs generated in CAD.

\section{Conclusions}

In this paper, we used two different morphing approaches to automatically batch morph FE models in the early digital development phase. The received FRF data was used to create metamodels describing the coherence between component hard point coordinates and characteristic FRF values. These metamodels were used to create a new component geometry that optimizes, for example, the transferred force at a specific frequency. This enables us to shift NVH optimization to earlier design phases and automate the creation of design recommendations to modify FRFs.

The validation proved both morphing approaches suitable to create metamodels, leading the design process in the right direction. As the creation of control blocks is quite time-consuming, the Box Morphing approach is suited for simpler geometries or complex geometries that require detailed feature preservation. For faster investigations, the Direct Morphing approach proved to be working well and easy adoptable to new components. For both approaches, the geometry changes on a complex component are restricted to small displacements, which makes larger topology investigations impossible. This is why the presented approaches especially apply to the early digital development phase, when the rough topology design is already concluded, but the specific component design is yet to be completed.

The metamodels proved to be able to direct the design process in the right direction. After creating the morphing environments, the automatically performed FE calculation, necessary to generate the metamodels, is the most time-consuming process. Both morphing approaches hinted the design to a reduction of a specific FRF peak. The presented simpler metamodels like two dimensional polynomials are suitable for quick design choices without having to perform numerical optimizations. This can help the component designer to consider NVH demands more easily without the need to create new FE models or even produce hardware prototypes. This can prevent dead-end designs to be implemented much earlier. The more complex higher dimensional metamodels though enable holistic optimizations to conclude optimum geometry designs. Additionally, the metamodel is able to perform a robustness analysis to determine the influences of geometric component tolerances.

The presented study showed that simple metamodels are able to direct the design process in the early digital design phase without the need for numerical optimization. In this phase, while many parameters are still to be discussed, a hint for the direction of change could be more valuable than a specific optimized design. Nevertheless, the already simulated data enables an optimization without any additional simulation effort. 
The used metamodels can still receive improvement for more complex problems. For example, instead of polynomials, we could use moving least squares or Kriging approaches, or even machine learning algorithms like neural networks.

The NVH optimization presented in this paper is the first step in a holistic optimization of the complete system, in our case the full vehicle. We can deploy the modified component into the full transfer path from road to passenger ear. The optimization of the sound pressure level is possible in a similar manner, as presented here. Kinematic hard points can serve as input for metamodels that predict the sound pressure level in the passenger cabin. The component and system designers obtain an easy to use tool to consider NVH demands in the early, digital design phase.

Author Contributions: Conceptualization, T.v.W., M.L. and F.G.; methodology, T.v.W. and M.L.; software, T.v.W. and M.L.; validation, T.v.W. and M.L.; writing-original draft preparation, T.v.W. and M.L.; writing一review and editing, T.v.W., M.L. and F.G.; visualization, T.v.W.; supervision, F.G.; project administration, T.v.W. All authors have read and agreed to the published version of the manuscript.

Funding: This research received funding by Mercedes-Benz AG.

Acknowledgments: The authors thank the Mercedes-Benz AG with the department NVH road noise, especially Ernst Prescha, Stéphanie Anthoine, and Wibke Lommatzsch for their support. Additionally, the authors would like to thank the members from the team and department for always being available to discuss the ongoing research. The authors thank Achim Winandi from the Karlsruhe Institute of Technology for valuable input and support.

Conflicts of Interest: The authors declare no conflict of interest. Timo von Wysocki and Michael Leupolz are from Mercedes-Benz AG, the company had no role in the design of the study; in the collection, analyses, or interpretation of data; in the writing of the manuscript, and in the decision to publish the results.

\section{Abbreviations}

The following abbreviations are used in this manuscript:

$\begin{array}{ll}\text { CAD } & \text { Computer Aided Design } \\ \text { CAE } & \text { Computer Aided Engineering } \\ \text { EFFD } & \text { Extended-Free-Form-Deformation } \\ \text { FE } & \text { Finite Element } \\ \text { FEM } & \text { Finite Element Method } \\ \text { FFD } & \text { Free-Form-Deformation } \\ \text { FRF } & \text { Frequency Response Function } \\ \text { NVH } & \text { Noise, Vibration and Harshness }\end{array}$

\section{References}

1. Mantovani, M. Rolling noise does not evoke an emotional response. ATZ Worldw. 2018, 120, $18-21$. [CrossRef]

2. Rambacher, C.; Ehrt, T.; Sell, H. Vibration optimisation of entire axles. ATZ Worldw. 2017, 119, 50-55. [CrossRef]

3. van der Auweraer, H.; van Langenhove, T.; Brughmans, M.; Bosmans, I.; Masri, N.; Donders, S. Application of mesh morphing technology in the concept phase of vehicle development. Int. J. Veh. Des. 2007, 43, 281-305. [CrossRef]

4. Helm, D.; Huf, A.; Zimmer, H.; Kondziella, R. Anforderungen in der frühen Phase der Gesamtfahrzeugauslegung. VDI-Berichte 2012, 2169, 21-40.

5. TrelleborgVibracoustic. Schwingungstechnik im Automobil: Grundlagen, Werkstoffe, Konstruktion, Berechnung und Anwendungen, 1st ed.; Vogel Business Media: Würzburg, Germany, 2015.

6. De Gaetano, G.; Mundo, D.; Vena, G.; Kroiss, M.; Cremers, L. A study on vehicle body concept modelling: Beam to joint connection and size optimization of beam-like structures. In Proceedings of the ISMA 2014 Including USD 2014, Leuven, Belgium, 15-17 September 2014; pp. 1653-1664.

7. Stigliano, G.; Mundo, D.; Donders, S.; Tamarozzi, T. Advanced Vehicle Body Concept Modeling Approach Using Reduced Models of Beams and Joints. In Proceedings of the ISMA 2010 Including USD 2012, Leuven, Belgium, 20-22 September 2010; pp. 4179-4190. 
8. Klein, B. FEM; Springer Fachmedien Wiesbaden: Wiesbaden, Germany, 2015. [CrossRef]

9. Fang, X.; Tan, K. Efficient Concept Design of Twist Beam Rear Axles. ATZ Worldw. 2015, 117, $24-29$. [CrossRef]

10. Staten, M.L.; Owen, S.J.; Shontz, S.M.; Salinger, A.G.; Coffey, T.S. A Comparison of Mesh Morphing Methods for 3D Shape Optimization. In Proceedings of the 20th International Meshing Roundtable; Quadros, W.R., Ed.; Springer: Berlin/Heidelberg, Germany, 2012; pp. 293-311. [CrossRef]

11. Alexa, M. Recent Advances in Mesh Morphing. Comput. Graph. Forum 2002, 21, 173-198. [CrossRef]

12. Nunes, R.F.; Will, J.; Bayer, V.; Karthik, C. Robustness Evaluation of Brake Systems Concerned to Squeal Noise Problem; Weimar Optimization and Stochastic Days 6; Dynardo GmbH: Weimar, Germany, 2009.

13. Nunes, R.F.; Stump, O.; Wolff, S. Use of Random Fields to Characterize Brake Pad Surface Uncertainties; Weimar Optimization and Stochastic Days 12; Dynardo GmbH: Weimar, Germany, 2015.

14. Moroncini, A.; Cremers, L.; Baldanzini, N. Car body concept modeling for NVH optimization in the early design phase at BMW: A critical review and new advanced solutions. In Proceedings of the International Conference on Noise and Vibration Engineering ISMA 2012 Including USD 2012, Leuven, Belgium, 17-19 September 2012; pp. 3809-3824.

15. Siemens AG. NX NASTRAN; Siemens AG: München, Germany, 2020.

16. Wolff, S. Random Fields and Field Meta Models: Correlation Analysis in Time and Space. RDO-J. 2016, 2-8.

17. Maressa, A.; Pluymers, B.; Desmet, W.; Donders, S. Optimization methodologies based on structural modification analysis in automotive NVH design. In Proceedings of ICSV 18; Crocker, M., Ed.; International Institute of Acoustics \& Vibration: Auburn, AL, USA, 2011; Volume 2, pp. 1780-1787.

18. Calì, M.; Oliveri, S.M.; Evangelos Biancolini, M.; Sequenzia, G. An Integrated Approach for Shape Optimization with Mesh-Morphing. In Advances on Mechanics, Design Engineering and Manufacturing II; Cavas-Martínez, F., Eynard, B., Fernández Cañavate, F.J., Fernández-Pacheco, D.G., Morer, P., Nigrelli, V., Eds.; Springer: Cham, Switzerland, 2018; pp. 311-322. [CrossRef]

19. Donders, S.; Takahashi, Y.; Hadjit, R.; van Langenhove, T.; Brughmans, M.; van Genechten, B.; Desmet, W. A reduced beam and joint concept modeling approach to optimize global vehicle body dynamics. Finite Elem. Anal. Des. 2009, 45, 439-455. [CrossRef]

20. Siebertz, K.; van Bebber, D.; Hochkirchen, T. Statistische Versuchsplanung; Springer: Berlin/Heidelberg, Germany, 2017. [CrossRef]

21. Von Wysocki, T.; Chahkar, J.; Gauterin, F. Small Changes in Vehicle Suspension Layouts Could Reduce Interior Road Noise. Vehicles 2020, 2, 18-34. [CrossRef]

22. Hemsley, R. Interpolation on a Magnetic Field; Technical Report; Bristol University: Bristol, UK, 2009.

23. Stevens, R. Discrete Sibson (Natural Neighbor) Interpolation, Naturalneighbor: 0.2.1. 2018. Available online: https:/ / pypi.org/project/naturalneighbor/ (accessed on 22 April 2020).

24. Python Software Foundation. Python; Python Software Foundation: Wilmington, DE, USA, 2020.

25. SciPy. scipy.interpolate.RegularGridInterpolator. Available online: https://docs.scipy.org/doc/scipy/ reference/generated/scipy.interpolate.RegularGridInterpolator.html (accessed on 2 November 2020).

26. Sederberg, T.W.; Parry, S.R. Free-form deformation of solid geometric models. In Proceedings of the 13th Annual Conference on Computer Graphics and Interactive Techniques-SIGGRAPH '86; Evans, D.C., Athay, R.J., Eds.; ACM Press: New York, NY, USA, 1986; pp. 151-160. [CrossRef]

27. Coquillart, S. Extended free-form deformation: A sculpturing tool for 3D geometric modeling. In Proceedings of the 17th Annual Conference on Computer Graphics and Interactive Techniques—SIGGRAPH '90; Baskett, F., Ed.; ACM Press: New York, NY, USA, 1990; pp. 187-196. [CrossRef]

28. Sieger, D.; Menzel, S.; Botsch, M. On Shape Deformation Techniques for Simulation-Based Design Optimization. In New Challenges in Grid Generation and Adaptivity for Scientific Computing; Perotto, S., Formaggia, L., Eds.; Springer International Publishing: Cham, Switzerland, 2015; pp. 281-303. [CrossRef]

29. Griessmair, J.; Purgathofer, W. Deformation of solids with trivariate B-splines. In Proceedings of Eurographics; Eurographics Association: Geneve, Switzerland, 1989; Volume 89, pp. 137-148. [CrossRef]

30. BETA CAE Systems. ANSA Pre Processor. Available online: https://www.beta-cae.com/ansa.htm (accessed on 2 November 2020). 
31. Dynardo (Dynamic Software and Engineering) GmbH. Optislang; Dynardo GmbH: Weimar, Germany, 2020.

32. Montgomery, D.C.; Runger, G.C. Applied Statistics and Probability for Engineers, 3rd ed.; Wiley: New York, NY, USA, 2003.

(C) 2020 by the authors. Licensee MDPI, Basel, Switzerland. This article is an open access article distributed under the terms and conditions of the Creative Commons Attribution (CC BY) license (http://creativecommons.org/licenses/by/4.0/). 